\title{
EXPERIÊNCIA INICIAL EM TRANSPLANTE DE INTESTINO DELGADO EM HOSPITAL ESCOLA DE SÃO JOSÉ DO RIO PRETO.
}

\author{
Report on the initial experience in small bowel transplantation at São José do Rio Preto Medical \\ School Hospital
}

Renato Ferreira da Silva ${ }^{1}$, Andréia Cristina de Paula 1 , Paulo César Arroyo Júnior ${ }^{1}$, Adriano Miziara Gonzales ${ }^{2}$ Julio Sérgio
Marchine ${ }^{3}$, William José Duca ${ }^{1}$, Márcia Fumiê Rocha ${ }^{1}$, Helen de Felício ${ }^{1}$, Mario Abbud-Filho ${ }^{4}$ e Rita de Cássia da Silva ${ }^{1}$

\section{RESUMO}

Falência intestinal é a incapacidade do indivíduo de manter seu suporte nutricional e hidroeletrolítico por via digestiva. É decorrente de grandes enterectomias ou de doenças onde o intestino é incapaz de absorver adequadamente fluídos e nutrientes. Pacientes com falência intestinal associada à síndrome do intestino curto, outras doenças funcionais com má absorção e complicações da nutrição parenteral total (sepse de repetição e trombose de um ou mais acessos venosos profundos) são candidatos a transplante de intestino delgado, que pode ser isolado, associado ao de fígado ou multivisceral.

Em nossa instituição, foram realizados três transplantes de intestino delgado isolado, que serão relatados neste artigo para descrever nossa experiência inicial nessa modalidade de transplante.

Descritores: Intestino Delgado; Síndrome do Intestino Curto; Transplante de Órgãos.

\footnotetext{
Instituições:

1 Unidade de Cirurgia do Fígado e Transplantes Gastrintestinais do Hospital de Base da Faculdade de Medicina de São José do Rio Preto - FUNFARME/ FAMERP - São José do Rio Preto / SP - Brasil

2 Serviço de Transplante do Aparelho Digestivo do Hospital São Paulo da Universidade Federal de São Paulo - UNIFESP - São Paulo / SP - Brasil

${ }^{3}$ Divisão de Nutrologia do Departamento de Clínica Médica da Faculdade de Medicina de Ribeirão Preto - Universidade de São Paulo - USP / Ribeirão Preto / SP - Brasil

${ }^{4}$ Centro de Transplantes de Órgãos e Tecidos - CINTRANS da Faculdade Medicina de São José do Rio Preto - FUNFARME/FAMERP - São José do Rio Preto / SP - Brasil
}

\section{Correspondência:}

Prof. Dr. Renato Ferreira da Silva.

Unidade de Cirurgia do Fígado e Transplantes Gastrintestinais do Hospital de Base da Faculdade de Medicina de São José do Rio Preto - FUNFARME/ FAMERP.

Av. Brigadeiro Faria Lima, 5416 - CEP: 15090-090 - São José do Rio Preto / SP - Brasil Fone: (17) 32015000

E-mail: renatofsbr@gmail.com /wjduca@ig.com.br

\section{INTRODUÇÃO}

A incapacidade do indivíduo em manter seu suporte nutricional e hidroeletrolítico por via digestiva é denominada falência intestinal. ${ }^{1}$ É fator determinante na síndrome do intestino curto (SIC), que ocorre quando, congenitamente ou após cirurgias, o paciente tem menos de $200 \mathrm{~cm}$ de intestino delgado. ${ }^{2,3}$ A SIC é caracterizada por perda ponderal, desnutrição, esteatorréia e diarréia ácida. Pode estar associada à colelitíase, nefrolitíase e hipersecreção ácida gástrica. Entre as causas de SIC destacam-se: extensas enterectomias devido à trombose ou embolia da artéria mesentérica, doença de Crohn, volvo intestinal, trauma, enterocolite necrotizante, gastrosquise, tumores intestinais (desmóide, gastrinoma e polipose familiar), absorção intestinal insuficiente e neuropatias viscerais. ${ }^{3}$

Pacientes com SIC e falência intestinal são candidatos à nutrição parenteral total - NPT. ${ }^{2}$ Nos EUA existem 30.000 pessoas dependentes de NPT, e estima-se que de 2 a 3/1.000.000 de habitante/ ano, apresentarão falência intestinal. ${ }^{1}$

O transplante de intestino delgado (TID) é o tratamento de escolha para pacientes com SIC que desenvolvem complicações decorrentes da NPT crônica como: infecções dos cateteres venosos centrais e sepsis de repetição, bem como trombose de um ou mais acessos venosos profundos., ${ }^{1,4-6}$ Tais morbidades, associadas à baixa qualidade de vida, internações frequentes ou permanentes, distúrbios psiquiátricos e custo elevado do tratamento respondem por mortalidade de 5 a $25 \%$ ao ano..$^{7-11}$

Existem três categorias de transplante de intestino delgado: isolado, associado ao fígado e multivisceral. A seleção do doador é baseada 
na compatibilidade ABO, peso e sorologia para citomegalovírus (CMV). Opta-se preferencialmente por doador CMV negativo, para evitar riscos de CMV grave e persistente no receptor. ${ }^{1}$

De acordo com o Registro Internacional de Transplantes Intestinais, foram realizados mais de 1000 transplantes no mundo com boa função do enxerto, restabelecimento da nutrição enteral e melhor qualidade de vida do receptor, além de evidenciar que a morbimortalidade atual é semelhante a da NPT permanente. ${ }^{1,12}$

Os primeiros relatos de realização do transplante de intestino em caráter experimental datam de 1959, quando Lillehei e col. publicaram o sucesso da retirada, preservação e reinserção do intestino em cães. Em 1968, no Boston Floating Hospital, Deterling e Fisher realizaram os dois primeiros transplantes de intestino delgado isolado do mundo, mas com óbito dos receptores. Okumura e col. realizaram em 1968 os dois primeiros transplantes de intestino delgado no Brasil, mas os receptores evoluíram a óbito. No ano de 2000, na Santa Casa de São Paulo, Iasi e Soler realizaram o primeiro transplante pediátrico do país em uma criança de dois anos, e obtiveram sobrevida de 100 dias. ${ }^{13,14} \mathrm{Em}$ nossa instituição, foram realizados três transplantes intestinais, sendo o primeiro em 2004.

No presente artigo, será descrita nossa experiência inicial com transplante de intestino delgado isolado, através dos relatos dos três casos.

\section{RELATOS DE CASOS}

\section{CASO 1}

Paciente MLP, sexo feminino, 34 anos, foi submetida em 2001 a parto por cesariana devido a óbito fetal, quando foi constatada extensa necrose intestinal acometendo desde a terceira porção duodenal até o cólon transverso. O diagnóstico de Síndrome do Anticorpo Antifosfolípide foi estabelecido, baseado na tríade: positividade do anticorpo anticardiolipina, óbito fetal e trombose mesentérica. A paciente permaneceu em NPT permanente, e apresentou complicações graves como: infecções de corrente sanguínea, dois episódios de endocardite e trombose de dois acessos venosos profundos. A avaliação pré-operatória foi normal, exceto pelo achado de esteatose hepática macrogoticular grau IV, evidenciada na biópsia hepática e avaliação nutricional evidenciando desnutrição calórica grave e protéica moderada, com índice de massa corporal (IMC) de $15,7 \mathrm{Kg} / \mathrm{m}^{2}$. Foi submetida a transplante de intestino delgado isolado em 26 de maio de 2004, evoluindo no pós-operatório sem intercorrências. A terapêutica imunossupressora endovenosa foi realizada com basiliximab, inibidor de calcineurina (tacrolimus) e corticóide. No quinto dia pós-operatório foi realizada colonoscopia com magnificação, que mostrou motilidade intestinal normal e alargamento das vilosidades intestinais em áreas circunscritas, com eritema moderado. Foram realizadas biópsias nos locais com eritema, e o exame anatomopatológico evidenciou rejeição leve.

Foram ajustadas as doses dos imunossupressores, e o nível sérico de tacrolimus estava ideal (29 ng/dL). Após o exame, a paciente evoluiu com taquicardia progressiva, seguida de parada cardiorrespiratória. A necropsia indicou infarto agudo do miocárdio como causa da morte.

\section{CASO 2}

Paciente JAV, sexo masculino, 66 anos, com diagnóstico de hipertensão arterial sistêmica, fibrilação atrial crônica, doença pulmonar obstrutiva crônica e depressão. Havia sido submetido em 2005 à ressecção intestinal extensa de intestino delgado, devido à isquemia mesentérica ocasionada por oclusão embólica da artéria mesentérica superior. Restaram apenas $10 \mathrm{~cm}$ de jejuno, em jejunostomia terminal, $5 \mathrm{~cm}$ de íleo terminal em ileostomia e todo cólon. Evoluiu com SIC, desnutrição protéica - calórica grave, com IMC de 18,1 Kg/m². Era dependente de NPT permanente e desenvolveu complicações dos cateteres venosos centrais, como: sepsis de repetição e trombose de dois acessos venosos profundos. A avaliação pré-operatória foi normal, exceto por disfunção contrátil de ventrículo esquerdo, hipertensão pulmonar discreta e fração de ejeção de 52\% no ecocardiograma; colestase moderada, associada à reação portal biliar discreta, siderose acentuada de grau IV, esteatohepatite discreta e esteatose microvesicular grau I na biópsia hepática. Foi submetido a transplante de intestino delgado isolado em 11 de dezembro de 2005, evoluindo com síndrome compartimental abdominal e necessidade de descompressão com peritoniostomia no pós-operatório imediato. Mantida terapêutica imunossupressora endovenosa com basiliximab, tacrolimus e corticóide. Apresentou evolução favorável no pós-operatório, tendo sido realizados curativos abdominais e aproximação cirúrgica gradual da parede abdominal a cada dois dias. No quinto pós-operatório, a colonoscopia com magnificação evidenciou motilidade intestinal normal e áreas de hiperemia discreta nas vilosidades, que passaram por biópsia. $\mathrm{O}$ exame anatomopatológico mostrou rejeição moderada e a dosagem dos imunossupressores foi ajustada, sendo o nível sérico de tacrolimus elevado (acima de $30 \mathrm{ng} / \mathrm{dL}$ ). No décimo terceiro pós-operatório evoluiu com choque séptico, sendo suspenso o tacrolimus, retirado o cateter venoso central e realizadas hemoculturas e culturas da ponta do cateter, nas quais cresceram colônias de Staphylococcus aureus. Foi introduzida a antibioticoterapia adequada, porém, o paciente evoluiu com disfunção de múltiplos órgãos e óbito no décimo oitavo dia pós-operatório.

\section{CASO 3}

O paciente PLA, sexo masculino, 49 anos apresentou isquemia mesentérica em 2004, devido a trombose da artéria mesentérica superior, sendo submetido à ressecção intestinal extensa. Tinha 30 cm de jejuno em todo o cólon, com trânsito alimentar reconstruído. Durante investigação, não foi possível diagnosticar a causa da trombose da artéria mesentérica superior. Era dependente de NPT para suplementar a nutrição enteral. Apresentava diarréia ácida e esteatorréia, controladas com loperamida. Tinha IMC de 22,7 Kg/ $\mathrm{m}^{2}$ e desnutrição protéico-calórica leve. Desenvolveu infecções de corrente sanguínea, dois episódios de sepse relacionados ao cateter venoso central e trombose de tronco braquicefálico e dois acessos venosos profundos. Após período sem infecções, foi submetido à colocação de portocath e esquema de NPT hospitalar apenas quatro vezes por semana. A avaliação pré-operatória foi satisfatória, exceto pela biópsia hepática mostrar esteatohepatite discreta com septos porta-porta e fibrose perisinusoidal centro-lobular, estádio estrutural F2 e esteatose macrovesicular discreta grau I. O transplante de intestino delgado foi realizado em 21 de fevereiro de 2006. O paciente evoluiu com isquemia e perda do enxerto no pós-operatório imediato, devido à trombose de artéria mesentérica superior do doador. Houve 
sobrevida do receptor, que permaneceu com seu trânsito alimentar reconstruído e evoluiu com readaptação intestinal, sendo necessária NPT hospitalar durante três vezes por semana.

\section{DISCUSSÃO}

Recentemente, a United Nation Organ Sharing (UNOS) mostrou que no ano de 2004 foram realizados 145 transplantes de intestino delgado, com 77,4\% de função do enxerto após o primeiro ano, e, após cinco anos de transplante, a sobrevivência é inferior a 50\%. ${ }^{15}$

Em 10\% dos pacientes a função do enxerto é parcial, e em $12 \%$ o enxerto é removido. ${ }^{1}$ As causas mais comuns de remoção do enxerto são rejeição (57\%), trombose, isquemia ou sangramento (23\%), falência de múltiplos órgãos (4\%), linfoma (2\%), sepse (1\%), e outras (4\%). O intestino é extremamente susceptível à isquemiareperfusão, fatores que determinam o sucesso e a sobrevida do enxerto. A agregação plaquetária aumenta após reperfusão do enxerto, resultando em alto risco de trombose vascular, ${ }^{16}$ como ocorreu no CASO 3, sendo necessária remoção do enxerto.

Outra complicação descrita é a síndrome compartimental abdominal e necessidade de peritoniostomia pós-transplante, como relatado no CASO 2. Esse fato ocorre em pacientes com retração da aponeurose abdominal. Na literatura, há opções cirúrgicas como peritoniostomia, uso de telas sintéticas ou aproximação apenas da pele. Entretanto, tais alternativas aumentam o risco de infecção ou fístulas, quando ocorre a adesão entre as alças intestinais e as telas sintéticas. ${ }^{17}$ Talvez a melhor opção nesse caso fosse a redução do enxerto que permitisse o fechamento do abdome.
Para diagnóstico macroscópico precoce de áreas intestinais sugestivas de rejeição, propiciando um reajuste das drogas imunossupressoras e evitando complicações, a colonoscopia com magnificação é o exame de escolha. Associada à biópsia desses locais, a literatura mostra alto índice de correlação entre macroscopia e resultados anatomopatológicos mostrando rejeição..$^{18}$ Os achados descritos nos CASOS 1 e 2 comprovam tal afirmação.

As principais causas de óbito são: sepse (49\%), falência de múltiplos órgãos (26\%), rejeição (10\%), linfoma e complicações técnicas (8\%) e outras (4\%). Nas fases iniciais pós-transplante há necessidade de manter altos níveis de imunossupressores para evitar a rejeição; porém, pode ocorrer disfunção renal, hipertensão arterial e suscetibilidade a infecções até fatais. ${ }^{1}$ A causa de óbito no CASO 2 comprova os resultados da literatura. Já o CASO 1 chama a atenção para a reativação da Síndrome do Anticorpo Antifosfolípide como uma potencial limitação para o sucesso do transplante intestinal. Não encontramos outro caso de TID na literatura em língua inglesa de paciente com a Síndrome do Anticorpo Antifosfolipede.

Os três casos relatados apresentaram complicações que confirmam as descritas na literatura. Também reafirmam que, atualmente, o transplante de intestino delgado é uma alternativa real para o tratamento de falência intestinal permanente. Pesquisas para melhorar a terapêutica imunossupressora, prevenção das infecções, aprimoramento da técnica cirúrgica, a seleção adequada dos doadores e receptores são fatores essenciais e vitais para o sucesso e aumento da sobrevida desse transplante.

\section{ABSTRACT}

Intestinal failure is the patient's inability to keep his or her hidroelectrolitic and nutritional support by the digestive way, arisen out of massive enterectomy or diseases in which the bowel is unable of appropriately absorb fluids and nutrients. Patients with intestinal failure associated to short bowel syndrome and other functional diseases that present malabsorption and total parenteral nutrition and related complications (recurrent sepsis and thrombosis of one or more deep venous accesses) are candidates for small bowel transplant, which can be an isolated small bowel transplant, a combined liver/small bowel transplant or a multivisceral one.

In our institution, three isolated small bowel transplants were carried out, and they will be reported in this article seeking to describe our initial experience in such transplant.

Keywords: Intestine, Small; Short Bowel Syndrome; Organ Transplantation

\section{REFERÊNCIAS}

1. Galvão FHF, Waitzberg DL, Bachella T, Gama-Rodrigues J, Machado MCC. Transplante de intestino delgado. Arq Gastroenterol. 2003;40:118-25.

2. American Gastroenterological Association on Short Bowel Syndrome. Gastroenterology. 2003;124:1105-10.

3. Kemp R, Correia RB, Sankarankutty AK, Santos JS, Módena JLP, Mente ED, et al. Liver disease associated with intestinal failure in the small bowel syndrome. Acta Cir Bras. 2006;21 Supl 1:67-71.

4. Pinna AD, Weppler D, Nery J, Ruiz P, Kato T, Khan F, et al. Intestinal transplantation at the University of Miami - five years of experience. Transplant Proc. 2000;32:1226-7.
5. Beckurts KTE, Stippel D, Scheimer K, Schäfer H, Benz C, Dienes HP, et al. First case of isolated small bowel transplantation at the University Cologne: rejectionfree course under quadruple immunossupression and endoluminal monitoring with video-capsule. Transplant Proc. 2004;36:340-2.

6. Giraldo M, Martin D, Colangelo J, Bueno J, Reyes J, Fung JJ, et al. Intestinal transplantation for patients with short gut syndrome and hypercoagulable states. Transplant Proc. 2000;32:1223-4.

7. Abu-Elmagd K, Reyes J, Fung JJ, Mazariegos G, Bueno J, Janov C, et al. Evolution of clinical intestinal transplantation improved outcome and cost effectiveness. Transplant Proc. 1999;31:582-4.

8. DiMartini A, Rovera GM, Graham TO, Furukawa H, Todo S, Funovita M, et al. Quality of life after small intestinal transplantation and among home parenteral nutrition patients. JPEN J Parent Enteral Nutr. 1998;22:357-62. 
9. Dionigi P, Alessini M, Ferrazi A. Irreversible intestinal failure, nutrition support, and small bowel transplantation. Nutrition. 2001;17:747-50.

10. Galvão FHF, Ye Q, Doughton S, Murase M, Todo S, Waitzberg DL, et al. Experimental animal model of graft-versus host disease (GVHD) after small bowel transplantation: characteristics of the model and application for developing treatment strategies. Transplant Proc. 1997;29:700.

11. Howard L, Hassan N. Home parenteral nutrition. 25 years later. Gastroenterol Clin North Am. 1998;27:481-512.

12. The Intestinal transplant Registry. http://www.intestinaltransplant.org, 2006.

13. Okumura M, Mester $\mathrm{M}$. The coming of age os small bowel transplantation in humans: a historical perspective. Transplant Proc. 1992;24:1242.

14. Iasi M, Soler WV, Iasi MSF, Favero SSG, Cordovani NTB, Rittes JC, et al. Transplante de intestino é uma realidade! J Brás Transpl. 2001;3:11-3.
15. United Nation Organ Sharing (UNOS). http://unos.org/data/about/viewdatareports. asp, 2000-2006.

16. Siniscalchi A, Spedicato S, Lauro A, Pinna AD, Cuchetti A, Dazzi A, et al. Intraoperative coagulation evaluation of ischemia-reperfusion injury in small bowel transplantation: a way to explore. Transplant Proc. 2006;38:820-2.

17. Asham E, Ukinis MF, Rastellini C, Elias G, Cicalese L. Acellular dermal matrix provides a good option for abdominal wall closure following small bowel transplantation: a case report. Transplant Proc. 2006;38:1770-1.

18. Misra MV, Bhattacharya K, Nompleggi DJ, Uknis ME, Rastellini C, Cicalese L. Magnificatrion Endoscopy as a rellable tool for the early diagnosis of rejection in living related small bowel transplants: a case report. Transplant Proc. 2006;38:1738-9. 